\title{
Estudo retrospectivo das curvaturas da coluna vertebral e do posicionamento pélvico em imagens radiográficas
}

\author{
Paola Trindade Hahn*, Cintia Bonilha Ulguim*, Ana Fátima Viero
}

Badaraó**

\begin{abstract}
RESUMO: Este estudo caracteriza-se em uma pesquisa documental retrospectiva, do tipo descritiva, com o objetivo de verificar o posicionamento da pelve e das curvaturas da coluna vertebral. Foram analisadas 104 imagens radiográficas de perfil, em adultos de ambos os sexos.Verificou-se em centímetros o desnível pélvico e utilizou-se o método Cobb e a razão entre a distância e a altura para analisar as curvaturas da coluna. Os resultados apontam a prevalência de retroversão pélvica, com mediana de $-0,6$ (-1,5-0). Na coluna os segmentos da cervical e lombar apresentaram padrão de normalidade $\left(39,5^{\circ} \pm 13,9,46,89^{\circ} \pm 9,63\right)$ e a torácica apresentou padrão entre normal e hipercifose $\left(45,05^{\circ} \pm 9,83\right)$.
\end{abstract}

Descritores: Coluna vertebral; Pelve; Radiografia

\section{Retrospective analysis of the spine curvatures and pelvic positioning on} radiographic images

ABSTRACT: This study is characterized by a retrospective documentary research, descriptive, aiming to check the positioning of the pelvis and the curvature of the spine. 104 radiographs were analyzed in profile, in adults of both sexes. In a centimeter scale, the pelvic gap was verified and the Cobb method and the ratio between the distance and height were used to analyze the curvature of the spine. The results indicate the prevalence of pelvic retroversion, with a median of $-0.6(-1,5-0)$. In the column, the cervical and lumbar spine segments showed normal range $\left(39.5^{\circ} \pm 13.9,46.89^{\circ} \pm 9.63\right)$ and the thoracic segment showed a pattern between normal and hyperkyphosis $\left(45.05^{\circ} \pm 9.83\right)$.

Descriptors: Spine; Pelvis; Radiographic

* Graduada em Fisioterapeuta pela Universidade Federal de Santa Maria, Rio Grande do Sul, Brasil.

**Docente do Departamento de Fisioterapia e Reabilitação - Centro de Ciências da Saúde, Universidade Federal de Santa Maria, Rio Grande do Sul, Brasil. 


\section{Introdução}

A coluna vertebral tem seu posicionamento organizado de acordo com os estresses e as sobrecargas exercidas sobre o sistema musculoesquelético, em decorrência da ação gravitacional. A postura correta é a posição na qual um mínimo de estresse é aplicado em cada articulação, com resultado de um alinhamento do corpo com eficiência fisiológica e biomecânica. ${ }^{1,2}$

O equilíbrio fisiológico da coluna vertebral é mantido por meio de curvaturas no plano sagital - lordose cervical, lordose lombar, cifose dorsal e cifose sacra - que se estabelecem durante o desenvolvimento e crescimento do corpo. Os desvios posturais envolvem os segmentos da coluna vertebral, como causa ou conseqüência de alguma alteração na dinâmica funcional do corpo e, ao se instalarem, intervém, modificam e desorganizam toda harmonia corporal, acarretando uma série de comprometimentos posturais relacionados ao sistema musculoesquelético. ${ }^{3,4}$

As variações nos graus de curvatura permitem igualdade na distribuição de forças em toda a coluna vertebral. Qualquer alteração equilíbrio resulta em deformidades e adaptações na pelve. 0 mau alinhamento sagital apresenta-se como um exagero ou dimuinuição de curvaturas, ou seja, hiperlordose, hipercifose ou retificações. ${ }^{5,6}$

A pelve é o ponto de apoio mais estável do corpo humano. Ela equilibra a coluna vertebral e faz a transição de forças entre a coluna e os membros inferiores e vice-versa. As mudanças na sua posição, afetam diretamente 0 alinhamento da coluna. A curvatura lombar está equilibrada sobre a pélvis e pode variar sua inclinação de acordo com a relação que estabelecem entre $\mathrm{si}^{7,8}$. Diversos métodos, para analisar a postura corporal e as curvaturas da coluna vertebral são descritos na literatura, verificados nos planos sagital e frontal, de forma subjetiva ou objetiva. A radiografia é o exame que fornece maior objetividade e, por isso, é o mais utilizado ${ }^{9}$. As imagens radiográficas da coluna vertebral permitem a mensuração do alinhamento e das curvaturas da coluna, sendo o método Cobb o mais referenciado para realizar essas medidas. . $, 9,10,11^{-1}$

0 presente estudo tem como objetivo verificar o posicionamento da pelve e das curvaturas da coluna vertebral, descrever os graus e o coeficiente entre a distância e a altura dessas curvaturas por segmento cervical, torácico e lombar e, também, a medida do posicionamento pélvico, verificados em imagens radiográficas de perfil, em adultos de ambos os sexos.

\section{Metodologia}

O estudo caracteriza-se como uma pesquisa documental retrospectiva, com análise descritiva das medidas angulares e lineares das curvaturas da coluna vertebral e 0 posicionamento pélvico, analisado em radiografias panorâmicas no plano sagital em indivíduos adultos de ambos os sexos.

Saúde (Santa Maria), v.37, n.1, p. 31-42,

2011.

Estudo retrospectivo das curvaturas da coluna radiográficas

A proposta de estudo está de acordo com as Diretrizes e Normas Regulamentadoras de Pesquisas Envolvendo Seres Humanos, estabelecidas na Resolução 196/96, do Conselho Nacional de Saúde, obtendo aprovação do Comitê de Ética e Pesquisa da UFSM (CEPUFSM), com parecer $n^{0}$ 23081.007537/2010-23, de 10 de junho de 2010. 
A pesquisa foi realizada em um instituto de radiologia privado, da cidade de Santa Maria RS. No local, são realizados em torno de 100 exames radiológicos de coluna por mês, com tecnologia digital, armazenados em um banco de imagens. Os dados foram coletados durante os meses de junho a setembro de 2010 , buscando arquivos de imagens registrados no período de um ano, de março de 2009 a fevereiro de 2010.

A mensuração das imagens foi realizada pelas próprias autoras em um computador Apple Macintosh, utilizando o software Osirix 1.3.1 que transforma o computador em uma estação de trabalho PACS DICOM para processar e visualizar imagens médicas. A análise da variabilidade interobservador foi realizada entre as duas observadoras em 15\% $(n=15)$ da amostra.

Foram incluídas no estudo as imagens radiográficas panorâmicas da coluna vertebral em incidência anteroposterior e perfil, de indivíduos de ambos os sexos, na faixa etária de 18 a 50 anos. Foram excluídas as imagens com curvaturas escolióticas maiores que $10^{\circ} \mathrm{Cobb}$, as que não documentaram a totalidade da coluna - desde a primeira vértebra cervical (C1) até os ossos da pelve, e aquelas com sinais de cirurgia, doenças degenerativas e anomalias congênitas. A incidência anteroposterior foi verificada apenas para detectar as curvaturas escolióticas maiores de $10^{\circ} \mathrm{Coob}$, para a exclusão do estudo.

$\mathrm{Na}$ verificação das medidas das curvaturas da coluna vertebral, foram utilizados dois parâmetros: 1- o método Cobb, criado inicialmente para verificar a angulação das curvaturas escolióticas $^{12}$, que fornece informações por meio de linhas traçadas sobre o bordo superior da vértebra limite superior (VLS), que é a primeira vértebra do segmento analisado, e sob 0 bordo inferior da vértebra limite inferior (VLI), a última vértebra deste segmento. 0 ângulo resultante da intersecção entre as retas VLS e VLI denomina-se ângulo Cobb, conforme 0 exemplo da coluna lombar (Figura 1); 2- o coeficiente entre a distância e a altura do segmento vertebral, denominado por Souchard de razão de flecha. ${ }^{13} \mathrm{~A}$ altura $(\mathrm{H})$ é medida por uma linha vertical traçada do ângulo superior do corpo da VLS ao ângulo inferior do corpo da VLI. A distância (L) é formada pela perpendicular que vai desta linha ao meio da borda lateral da vértebra ápice. A divisão da distância pela altura (L/H) fornece a razão da flecha (Figura 2).

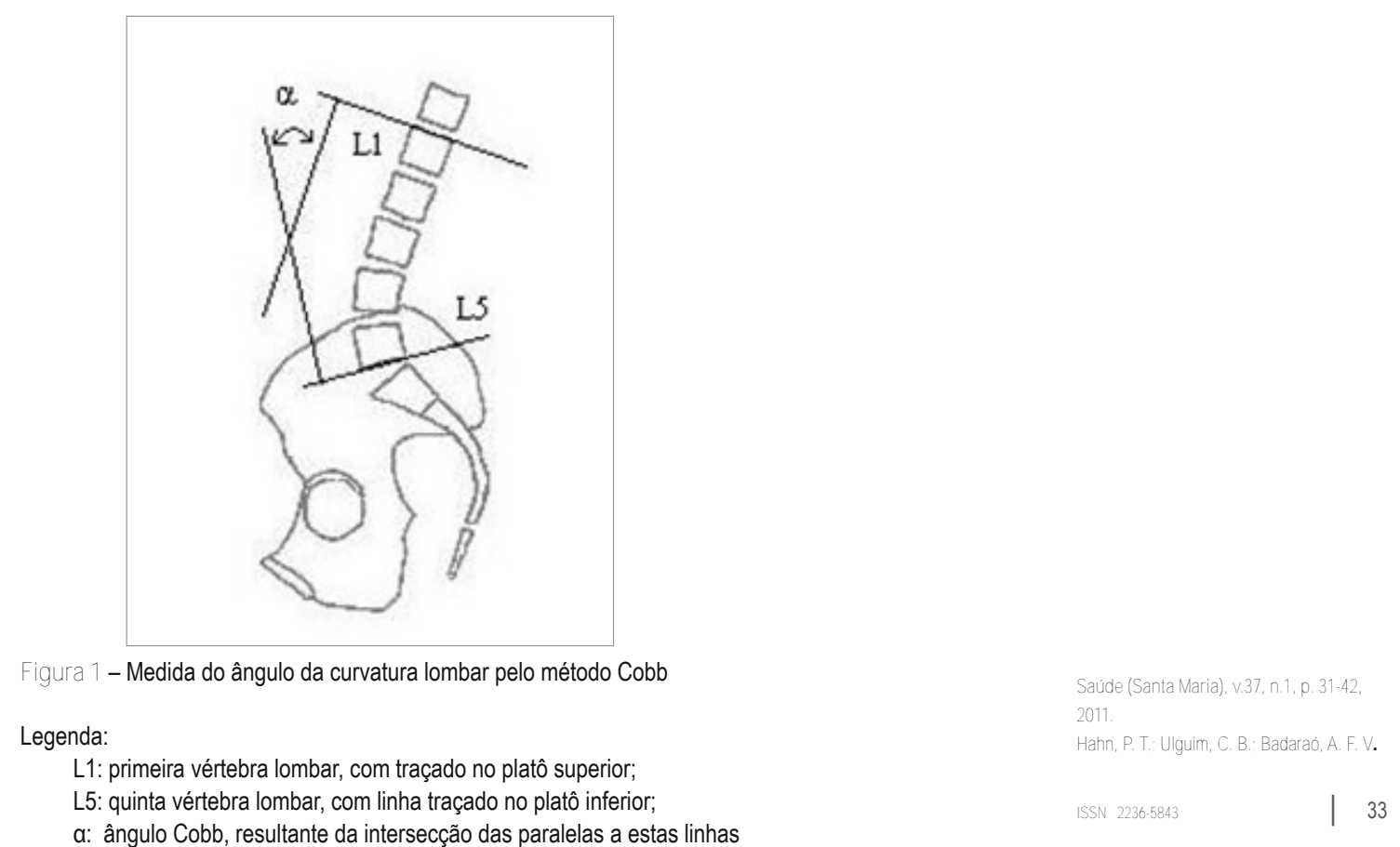




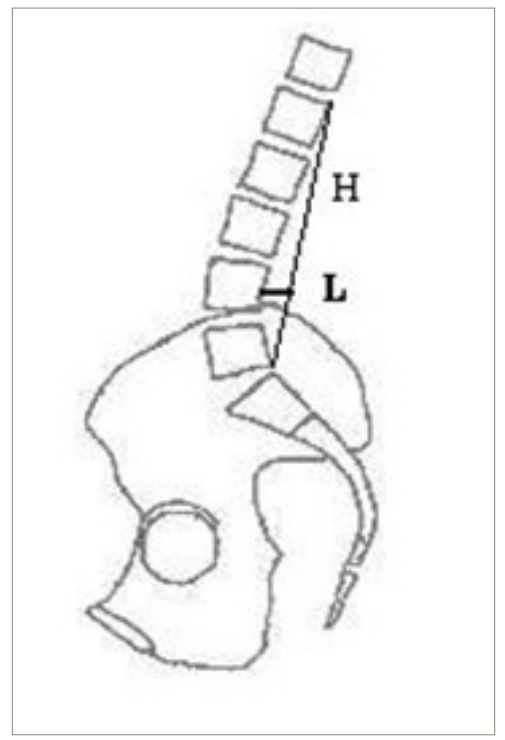

Figura 2 - Medida da altura e da distância para a curvatura da coluna (exemplo na lombar)

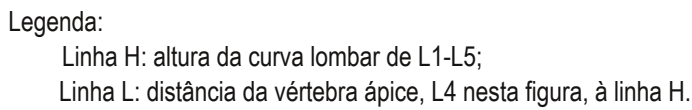

Os segmentos foram analisados separadamente, medindo-se as curvaturas: - cervical da primeira à sétima vértebra (C1-C7), suas subdivisões em cervical alta (C1-C2) e cervical baixa (C3-C7); - torácica (T1-T12) e, - lombar (L1-L5).

O alinhamento normal da pelve, do ponto de vista anatômico, é representado por uma linha horizontal que passa pela espinha ilíaca anterosuperior (EIAS) e pela espinha ilíaca posteroinferior (EIPI), no plano sagital, formando um ângulo de inclinação de zero grau ${ }^{14}$ (figura 3). Quando a linha não estiver horizontalizada, sua inclinação anterior caracteriza a anteversão pélvica, e a inclinação posterior, a retroversão pélvica.

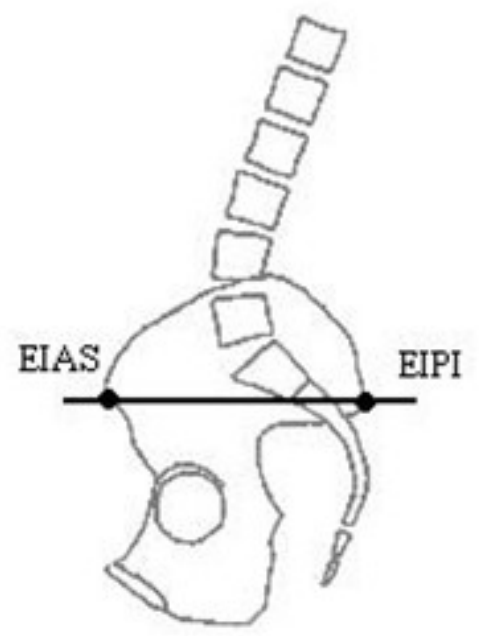

Saúde (Santa Maria), v.37, n.1, p. 31-42,

2011.

Estudo retrospectivo das curvaturas da coluna vertebral e do posicionamento pélvico em imagens

radiográficas

Figura 3 - Alinhamento pélvico ideal

Legenda:

EIAS - EIPI : Linha horizontal que passa pelas espinha ilíaca antero-superior e espinha ilíaca postero-inferior 
Neste estudo o posicionamento pélvico foi mensurado pela diferença de altura em centímetros da linha traçada entre EIAS e EIPI e foi considerado valor zero (0) para a ausência de inclinação, valor negativo (-) para inclinação posterior e valor positivo (+) para inclinação anterior. Foram considerados normais até um centímetro de anteversão pélvica na mulher e até um centímetro de retroversão no homem. ${ }^{15}$

Os dados foram analisados pelo Statistical Package for the Social Sciences (SPSS) versão 13.0 e estão expressos em média \pm desvio-padrão (DP) e mediana e amplitude interquartil (p25-75). As comparações foram realizadas após estratificação por faixa etária, em dois grupos, um com idades entre 18 e 30 anos (grupo 1) e outro de 31 a 50 anos (grupo 2), analisada pelo Teste $t$ de Student não pareado. A análise da variabilidade interobservador foi conduzida pelo Coeficiente de Correlação de Pearson. Considerado um nível de significância de $5 \%(p<0,05)$.

A análise dos resultados obtidos pela medida do ângulo de Cobb será realizada em comparação com estudos de outros autores. Para a análise dos resultados da razão entre a altura e a distancia das curvaturas, será utilizado como referência 0 estudo de Souchard ${ }^{13}$ sobre as escolioses, por não se encontrar textos com essa abordagem. 0 alinhamento pélvico foi interpretado pelos parâmetros indicados por Bienfait. ${ }^{14}$

\section{Resultados}

De acordo com os critérios de inclusão e exclusão estabelecidos, resultaram 104 imagens radiográficas para análise deste estudo. Destas, 17 imagens (16,4\%) foram de indivíduos do sexo masculino e $87(83,6 \%)$ do sexo feminino. Quanto à idade, 51 indivíduos estavam na faixa etária entre 18 e 30 anos e 53 entre 31 e 50 anos, resultando em uma média total de $31 \pm 9$ anos.

De acordo com o método cobb, encontraram-se os seguintes valores para as medidas das curvaturas da coluna: 1) coluna cervical 1a) alta(C1-C2): valores entre $9,02^{\circ}$ a $55,15^{\circ}$ Cobb, com média $32,70^{\circ} \pm 7,44 ; 1$ b) baixa(C3-C7): valores entre $0,48^{\circ}$ a $54,72^{\circ} \mathrm{Cobb}$ com mediana 8,7(4,1-12,8); 1c) total(C1-C7) valores entre $9,85^{\circ}$ a $84,90^{\circ} \mathrm{Cobb}$, com média $39,55^{\circ}$ $\pm 13,96$. 2) coluna torácica: as medidas variaram de $21,37^{\circ}$ a $66,21^{\circ}$ com média $45,05^{\circ} \pm$ 9,83. 3) coluna lombar: as medidas variaram de $15,11^{\circ}$ a $70,34^{\circ}$ com média $46,89^{\circ} \pm 9,63$.

As medidas da distância $(L)$ e da altura $(H)$ também foram verificadas nos segmentos da coluna vertebral. Calculou-se a razão dessas medidas $(\mathrm{L} / \mathrm{H})$, que estão demonstradas na tabela I. O alinhamento pélvico, observado pela horizontalidade entre EIAS e EIPS, apresentou valores entre $-4,31$ a 12,75cm com mediana de $-0,6$ (-1,5 - 0), na qual 9 imagens apresentaram as espinhas no mesmo plano, altura zero, 72 imagens apresentaram valores negativos e 23 apresentaram valores positivos. Analisados por gênero, no sexo feminino $(n=87) 8$ imagens apresentaram o alinhamento pélvico com valor igual a zero; $21 \mathrm{com}$ valores positivos e $58 \mathrm{com}$ valores negativos. Já, para as imagens dos indivíduos do sexo masculino ( $n=17$ ), uma imagem obteve resultado zero, duas foram com valores positivos e 14 com valores negativos.

A comparação dos grupos, analisados por faixa etária, apresentou diferença estatisticamente significativa entre as medidas do ângulo Cobb da cervical, altura da cervical e

Saúde (Santa Maria), v.37, n.1, p. 31-42, 2011.

Hahn, P. T.; Ulguim, C. B.; Badaraó, A. F. V. ISSN $2236-5843$ 
altura lombar $(p<0,05)$. 0 grupo 1, com idade entre 18-30 anos $(n=51)$, apresentou média de $36,4^{\circ} \pm 13,5$ para o cobb da cervical. Enquanto o grupo 2, com idade entre 31-50 anos, apresentou média de $42,5 \pm 13,8$.

Tabela 1 - Distribuição dos parâmetros distância $(L)$, altura $(H)$ e razão $L / H$ nos diferentes segmentos da coluna vertebral.

\begin{tabular}{ccccc}
\hline Segmento & Parâmetro & Mínimo & Máximo & Média/Median \\
\hline Cervical & Altura $(\mathrm{cm})$ & 9,1 & 17,3 & $11,83 \pm 1,48$ \\
& Distância $(\mathrm{cm})$ & 0 & 2,1 & $0,6(0-0,83)$ \\
& Razão L/H & 0 & 0,692 & $0(0-0,08)$ \\
\hline Torácica & Altura $(\mathrm{cm})$ & 22,6 & 31,9 & $27,1 \pm 6,04$ \\
& Distância $(\mathrm{cm})$ & 1,1 & 5,7 & $3,49 \pm 0.87$ \\
& Razão L/H & 0,022 & 0,230 & $0,120 \pm 0,03$ \\
& & & \\
\hline & Altura $(\mathrm{cm})$ & 13,5 & 19,1 & $16,37 \pm 1,04$ \\
& Distância $(\mathrm{cm})$ & 0 & 2,5 & $1,59 \pm 0,39$ \\
& Razão L/H & 0 & 0,163 & $0,09 \pm 0,02$ \\
\hline
\end{tabular}

Na medida da altura da cervical o grupo 1 obteve média de $12,1 \pm 1,1 \mathrm{~cm}$ e 0 grupo 2 média de $11,5 \pm 1,6 \mathrm{~cm}$. A altura do segmento lombar no grupo 1 apresentou-se com média de $16,1 \pm 0,9 \mathrm{~cm}$ e o grupo 2 média de $16,5 \pm 1,0 \mathrm{~cm}$.

Os resultados dos testes de confiabilidade interobservador foi avaliado pela análise de correlação entre avaliador 1 e 2 para todas as variáveis, mostrando boa confiabilidade entre as medidas interobservador (tabela 2). 
Tabela 2 - Resultado do teste de confiabilidade interobservador para os segmentos estudados ( $n=15)$

\begin{tabular}{ccc}
\hline Segmento & Parâmetro & Valor de r \\
\hline Cervical & Distância & 0,97 \\
& Altura & 0,90 \\
& Coeficiente L/H & 0,97 \\
Cervical alta & Ângulo Cobb & 0,97 \\
Cervical baixa & Ângulo Cobb (C1-C2) & 0,93 \\
& Ângulo Cobb (C3-C7) & 0,98 \\
Torácico & Distância & \\
& Altura & 0,93 \\
& Coeficiente L/H & 0,80 \\
& Ângulo Cobb & 0,95 \\
& Distância & 0,90 \\
Lombar & Altura & 0,91 \\
& Coeficiente L/H & 0,82 \\
& Ângulo Cobb & 0,88 \\
Pelve & Alinhamento & 0,97 \\
& & 0,97 \\
\hline
\end{tabular}

\section{Discussão}

As curvaturas sagitais da coluna vertebral apresentam grande variabilidade nos valores das medidas de indivíduos normais. ${ }^{16}$ Gore et al ${ }^{17}$ referem que, embora as radiografias apresentem anormalidades na coluna vertebral não necessariamente os indivíduos serão sintomáticos.

A medida da curvatura cervical, através do Método Cobb, é realizada em diferentes vértebras como VLS. Em estudos que analisaram a curvatura de C2-C7, Harrison e Donald ${ }^{18}$ observaram 400 radiografias cervicais e descreveram valores que variaram de $16,5-66^{\circ} \mathrm{com}$ média de $34^{\circ}$ para esta curva. Em outro estudo, Harrison et al19 encontraram valores médios de $26,8^{\circ}, 16,5^{\circ}$ e $12,7^{\circ}$, para indivíduos com dor aguda ( $n=52$ ), com dor crônica ( $n=70$ ) e assintomáticos $(n=71)$, respectivamente, indicando que os indivíduos com dor tinham a lordose cervical aumentada comparada com indivíduos normais.

No estudo de Castro e Lopes ${ }^{3}$, o valor médio para a lordose cervical, mensurado entre 0 platô superior de $\mathrm{C} 1$ e o platô inferior de $\mathrm{C} 7$, foi de $40^{\circ} \mathrm{Cobb}$. Hardacker et a $\mathrm{a}^{20}$ encontraram média total da lordose cervical de $40^{\circ} \pm 9,7^{\circ}$ observando que a maior inclinação da curva ocorreu no nível de C1-C2 enquanto que apenas 6 graus (15\%) ocorreram no nível mais baixo da curvatura.

Em nosso estudo, para a curvatura cervical (C1-C7), obteve-se a média de $39,5^{\circ} \pm 13,9$ de ângulos Cobb, padrão considerado dentro da normalidade de acordo com os estudos de Castro e Lopes ${ }^{3}$ e Hadacker et al ${ }^{20}$. A cervical alta apresentou angulação maior, com média de $\left(32,7^{\circ} \pm 7,4\right)$ do que a cervical baixa, na qual se observou uma distribuição irregular, com $8,7^{\circ}$ $(4,1-12,8)$ de mediana.

Saúde (Santa Maria), v.37, n.1, p. 31-42 2011. Hahn, P. T.; Ulguim, C. B.; Badaraó, A. F. V. ISSN 2236-5843 37 
Verificou-se na análise das curvaturas que as vértebras do segmento cervical comportamse de forma diferente dos demais segmentos. Estas se apresentaram em diversos graus de inclinações independentes, podendo apenas uma vértebra encontrar-se inclinada no plano sagital, aumentando o ângulo total da curvatura. Assim o posicionamento real fica mascarado, podendo-se ter um segmento retificado com uma angulação grande devido à inclinação de apenas uma vértebra, pois, de acordo com o método cobb utiliza-se somente a primeira e última vértebra do segmento.

Em revisão retrospectiva de 28 imagens radiograficas, realizada na University Hospital Spine Center, EUA - Sul da Califórnia, foi avaliada a curvatura torácica de T1-T12 e encontrouse a média de $46,5^{\circ} \pm 15,9$ com mínimo $8^{\circ}$ e máximo 89,90. ${ }^{21}$ Tayyab et al5 descrevem em seu estudo que 0 alinhamento normal da coluna torácica no plano sagital varia de $20^{\circ}$ a $40^{\circ}$.

No presente estudo, a curvatura da coluna torácica, obteve média de $45,05^{\circ} \pm 9,83$, com mínimo $21,37^{\circ}$ e máximo $66,21^{\circ}$, ângulos de Cobb, estando de acordo com o que Raymond et al ${ }^{21}$ apresenta como padrão de normalidade. Entretanto, ao se comparar com os estudos de Tayyab et al ${ }^{5}$, esses dados estão caracterizados como hipercifose.

A medida da lordose lombar, utilizando o método Cobb, pode ser analisada em diferentes níveis quanto ao posicionamento do primeiro traçado na vértebra (VLS) e do último traçado (VLI). Em uma análise radiográfica da lordose lombar, de 30 imagens, Harrison et al ${ }^{10}$, obteve

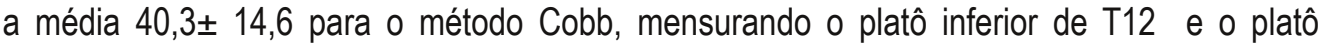
superior de primeira vértebra sacral (S1).

Gardocki et al22 encontram para a lordose lombar entre L1 e S1 a média de 50 graus com desvio padrão 14,3 e valores que variaram de $17,9^{\circ}$ a $89,5^{\circ}$. Em uma análise da angulação vertebral no plano sagital em uma população grega, o valor médio da lordose medindo-se de L1 a $L 5$ foi de $45,7^{\circ}$ obtidos dentro da faixa de $22^{\circ}$ a $54^{\circ}$, verificaram, ainda, que a lordose distal (L4-S1) representa $69 \%$ do total da lordose lombar. ${ }^{6}$

No presente estudo obteve-se a média angular de 46,89 $\pm 9,63$, com mínimo $15,11^{\circ} \mathrm{e}$ máximo $70,34^{\circ}$, para a curvatura da lombar (L1-L5), coincidindo com os estudos deste último autor.

A medida da razão $\mathrm{L} / \mathrm{H}$ apresenta vantagens em relação ao método Cobb por ser mais fácil de medir, reduzindo assim os erros de traçado, leva em consideração o comprimento das curvas e resulta em um dado preciso. Quando as vértebras limite possuem a mesma inclinação, a razão de flecha relaciona-se com o ângulo de Cobb, mas isto raramente ocorre, para transformá-la multiplica-se pelo coeficiente 458,5. ${ }^{13}$

Tabela 3 - Relação entre as médias de ângulo de Cobb e razão L/H (Souchard) ${ }^{13}$

\begin{tabular}{cc}
\hline Ângulo de Cobb & Razão L/H \\
\hline $30^{\circ}$ & 0,066 \\
$40^{\circ}$ & 0,088 \\
$50^{\circ}$ & 0,111 \\
\hline
\end{tabular}


Tabela 4 - Relação entre as médias de ângulo de Cobb e razão L/H encontradas no estudo

\begin{tabular}{cc}
\hline Ângulo de Cobb & Razão L/H \\
\hline $39,5^{\circ *}$ & 0,059 \\
$45,0^{\circ} \dagger$ & 0,121 \\
$46,8^{\circ} \ddagger$ & 0,096 \\
\hline
\end{tabular}

Legenda:

* Média encontrada no segmento cervical;

† Média encontrada no segmento torácico

‡ Média encontrada no segmento lombar.

De acordo com os dados apresentados na tabela 3 e 4 pode-se dizer que os valores obtidos aproximam-se com os descritos por Souchard. ${ }^{13}$ Observou-se que grande parte da amostra apresentou inclinações diferentes entre VLS e VLI. Supõem-se que este fator determinou a variação entre as tabelas.

Encontrou-se na análise dos dados do alinhamento pélvico que 24 indivíduos apresentam 0 posicionamento considerado normal, significando que nos homens as EIAS e EIPI encontram-se com distanciamento entre -1 e $0 \mathrm{~cm}$, e nas mulheres estão distanciando-se entre $0 \mathrm{e}+1 \mathrm{~cm}^{14}$. Destes, apenas 9 encontram-se com 0 alinhamento pélvico ideal, onde a linha horizontal passa pela EIAS e EIPI, com distância zero (0). A maior parte da amostra, $68 \%$ $(n=71)$, apresentou retroversão pélvica e apenas $8 \%(n=9)$ indivíduos apresentaram anteversão pélvica. $\mathrm{Na}$ análise por gênero, dos sujeitos do sexo masculino, $76 \%$ apresentaram a pelve em retroversão, $12 \%$ normal e 12\% em anteversão. No sexo feminino $67 \%$ apresentaram a pelve em retroversão, $25 \%$ normal e $8 \%$ em anteversão pélvica. $\mathrm{Na}$ literatura, não se encontrou referências sobre estudos em imagens radiográficas, para esse tipo de análise.

O envelhecimento fisiológico da coluna vertebral causa alterações das curvas sagitais normais resultando em uma estrutura não tão eficiente biomecanicamente, levando a um aumento da cifose torácica e diminuição da lordose lombar com a idade principalmente após a sétima década de vida. ${ }^{6,23,24} \mathrm{Em}$ nosso estudo não houve está relação quanto ao segmento torácico e lombar. Acredita-se que isso ocorreu devido à limitação da faixa etária em no máximo 50 anos, e este processo ocorrer de forma mais acentuada após os 70 anos. Foi encontrada diferença estatística significativa para as medidas do ângulo Cobb da cervical, altura cervical e altura lombar. 0 ângulo Cobb cervical diminui com a idade, enquanto a altura cervical e a altura lombar parecem aumentar em relação a esta variável.

\section{Conclusão}

Com base na análise das informações e os resultados obtidos neste trabalho, pode-se concluir que a medida das curvaturas da coluna vertebral, nas imagens radiográficas, pelo método Cobb, apresentou o padrão de normalidade para o segmento cervical e lombar. 0 segmento torácico caracterizou-se em padrão de normalidade à hipercifose. Em relação ao alinhamento da pelve verificou-se maior prevalência de retroversão pélvica.

Entretanto, na análise dos dados referentes à medida da altura e distância dos segmentos, esperava-se que quanto menor a altura da curvatura, maior seria a sua distância e, conseqüentemente, haveria um aumento da razão de L/H. Esta relação não foi verificada

Saúde (Santa Maria), v.37, n.1, p. 31-42, 2011. Hahn, P. T.; Ulguim, C. B.; Badaraó, A. F. V. ISSN 2236-5843 
neste estudo. Com os dados obtidos não foi possivvel determinar esta relação. Sugere-se novos estudos para determinação deste parâmetro e suas interferências.

Os estudos sobre este tema são descritos na literatura tendo como foco populações restritas, o que resulta em muitas variabilidades dessas medidas. No Brasil nenhum estudo foi encontrado, até o momento, relacionando radiografia com as curvaturas da coluna vertebral. Daí entende-se a importância destes resultados para subsidiar a continuidade desta investigação na população brasileira.

\section{Referências}

1. Palmer LM, Epler ME. Fundamentos das Técnicas de Avaliação Musculoesquelética. 2 ed. Rio de Janeiro: Guanabara Koogan, 2000.

2. Magee DJ. Disfunção Musculoesquelética. 3 ed. São Paulo: Manole, 2002.

3. Castro PCG, Lopes JAF. Avaliação computadorizada por fotografia digital, como recurso de avaliação na Reeducação Postural Global. Acta Fisiatr 2003: 10 (2): 83-88. Disponível em:< http://www.actafisiatrica.org.br/v1\%5Ccontrole/secure/Arquivos/AnexosArtigos/67C6A1E7CE56D3D 6FA748AB6D9AF3FD7/acta_vol_10_num_02_83_88.pdf>

4. Voutsinas AS, MacEwen GD. Sagittal profiles of the spine. Clin Orthop 1986: 210: $235-42$. Disponível em:<http://www.ncbi.nlm.nih.gov/pubmed/3757369>

5. Tayyab NA, Samartzis D, Altiok H, Shuff CE, Lubicky JP, Herman J, Khanna N. The Reliability and Diagnostic Value of Radiographic Criteria in Sagittal Spine Deformities: Comparison of the Vertebral Wedge Ratio to the Segmental Cobb Angle. Spine 2007 Jul 15: 32 (16): 451-459. Disponível em:< http://www.ncbi.nlm.nih.gov/pubmed/17632384>

6. Roussouly P, Nnadi C. Sagittal plane deformity: an overview of interpretation and management. Eur Spine J. 2010 Nov 19 (11):1824-36. Disponível em: < http://www.ncbi.nlm.nih.gov/pubmed/20567858>

7. Gonçalves GB, Pereira JS. Avaliação radiológica dos valores angulares das curvaturas lombolombar e lombosacra em adolescente. Act Fisistr 2008: 15 (2):92-95. Disponível em: $<$ <ttp://bases.bireme.br/cgibin/wxislind.exe/iah/online/?IsisScript=iah/iah.xis\&src=google\&base=LILA CS\&lang=p\&nextAction=Ink\&exprSearch=492518\&indexSearch=|D>

8.Legaye J, Duval-Beaupère G, Hecquet J, Marty C. Pelvic incidence: a fundamental pelvic parameter for three-dimensional regulation of spinal curves. Eur Spine J 1998: 7: 99-103. disponível em: <http://www.ncbi.nlm.nih.gov/pubmed/9629932>

9. Souza, FR, Ferreira, F. Narciso, FV.; Makhoul, CMB, Canto, RST, Barauna, MA. Avaliação da concavidade lombar pelo método radiográfico e pela cifolordometria. Rev Bras Fisioter 2009 marabr: $\quad 13 \quad$ (2): $103-109 . \quad$ Disponível <http://132.248.9.1:8991/hevila/Revistabrasileiradefisioterapia/2009/vol13/no2/2.pdf>

10. Harrison DE, Harrison DD, Cailliet R, Janik TJ, Holland B. Radiographic Analysis of Lumbar Lordosis: Centroid, Cobb, TRALL, and Harrison Posterior Tangent Methods. Spine 2001 Jun 1: 26: 235-242; ; disponível em: http://journals.Iww.com/spinejournal/Abstract/2001/06010/Radiographic_Analysis_of_Lumbar_Lordos is_.3.aspx>

11. Rosenfeldt MP, Harding IJ, Hauptfleisch JT, Fairbank J. A comparison of traditional protractor versus oxford Cobbmeter Radiographic Measurement. Spine 2005: 30 (4): 440-3. Disponível em: < http://www.springerlink.com/content/x224801747802731/>

12. Bradford DS, Lonstein JE, Moe JH, Ogivie JW, Winter RB. Escoliose e outras deformidades da coluna: o livro de moe. 2. ed. São Paulo: Santos; 1994. 
13. Souchard P, Ollier M. As escolioses - seu tratamento fisioterapêutico e ortopédico. São Paulo, SP: É realizações Ltda, 2001.

14. Bienfait, M. Os desequilibrios estáticos: fisiologia, patologia e tratamento fisioterápico. 5. ed. São Paulo: Summus, 1995.

15. Miranda, E. Bases da Anatomia e Cinesiologia. 2a ed. Rio de Janeiro: Sprint, 2000.

16. Damasceno, LHF, Catarin, SRG, Antônio DCA, Defino, HL. Lordose lombar: estudo dos valores angulares e da participação dos corpos vertebrais e discos intervertebrais. Acta Ortop Bras 2006 : 14(4):193-198. Disponível em:

http://www.scielo.br/scielo.php?pid=S141378522006000400003\&script=sci_arttext>

17. Gore DR, Sepic SB, Gardner GM. Roentgenographic findings of the cervical spine in asymptomatic people. Spine 1986 Jul-Aug:11(6): 521-4. Disponivel em:<http://journals.Iww.com/spinejournal/Abstract/1986/07000/Roentgenographic_Findings_of_the_

Cervical_Spine_in.3.aspx>

18. Harrison DD, Janik TJ, Troyanovich SJ, Holland B. Comparisons of lordotic cervical spine curvatures to a theoretical ideal model of the static sagittal cervical spine. Spine 1996 Mar 15: 21(6): 667-75 disponivel em: < http://www.ncbi.nlm.nih.gov/pubmed/8882687>

19. Harrison DD, Harrison DE, Janik TJ, Cailliet R, Ferrantelli JR, Haas JW, Holland B. Modeling of the sagittal cervical spine as a method to discriminate hypolordosis: results of elliptical and circular modeling in 72 asymptomatic subjects, 52 acute neck pain subjects, and 70 chronic neck pain subjects. $\quad$ Spine 2004 Nov 15: 29 (22): 2485-92. Disponível em: < http://www.ncbi.nlm.nih.gov/pubmed/15543059>

20. Hardacker, JW, Shuford, RF, BME; Capicotto, PN, Pryor, PW. Radiographic Standing Cervical Segmental Alignment in Adult Volunteers Without Neck Symptoms. Spine 1997 Jul 1: 22(13): 147280. Disponível em: < http://journals.Iww.com/spinejournal//Abstract/1997/07010/Radiographic_Standing_Cervical_Segment al_.9.aspx>

21. Raymond JG, Robert GW, Lytton AW. Measurements of lumbopelvic lordosis using the pelvic radius technique as it correlates with sagittal spinal balance and sacral translation. Phys Ther 2009 Nov : 89 (11): 1145-57. Disponível em: <http://www.ncbi.nlm.nih.gov/pmc/articles/PMC2899756/>

22. Gardocki RJ, Watkins RG, Williams LA. Measurements of lumbopelvic lordosis using the pelvic radius technique as it correlates with sagittal spinal balance and sacral translation. Spine 2001 Jun 1: 26(11): 235-42.

23.Korovessis PG, Stamatakis MV, Baikousis AG. Reciprocal angulation of vertebral bodies in the sagittal plane in an asymptomatic Greek population. Spine 1998 Mar 15: 23: 700-705. Disponível em

<http://journals.Iww.com/spinejournal/Abstract/1998/03150/Reciprocal_Angulation_of_Vertebral_Bodi es_in_the.10.aspx>

24. Pluijm S, Tromp AM, Smit.J.H, Deeg DJH, Lips P. Consequences of vertebral deformities in older men and women. J Bone Miner Res 2000: 15 (8): $1564-72$ Disponível em:< http://www.ncbi.nlm.nih.gov/pubmed/10934655>

\section{Paola Trindade Hahn}

Endereço para correspondência - Rua Venâncio Aires 2180/401 Bairro Centro, Santa Maria, Rio Grande do Sul, Brasil.

E-mail: paolinhath@hotmail.com

Recebido em 18 de abril de 2011.

Aceito em 18 de julho de 2011.

Saúde (Santa Maria), v.37, n.1, p. 31-42, 2011.

Hahn, P. T.; Ulguim, C. B.; Badaraó, A. F. V.

ISSN 2236-5843 